\title{
Remarks on $\gamma_{\hat{\Omega}}^{\star}$-open sets and minimal $\gamma_{\hat{\Omega} \text {-open sets }}^{\star}$
}

$$
\gamma_{\hat{\Omega}}^{\star}
$$

\section{K. Poorani, S. M. Meenarani, M. Anbuchelvi}

Abstract: Aim of this paper is to define $\gamma_{\hat{\Omega}}^{\star}$-open sets in a topological space and obtain their basic properties. Also, we

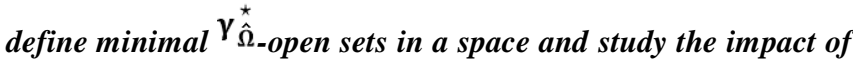
two minimal $\gamma_{\hat{\Omega}_{-}}^{\star}$ open sets in a space with $\hat{\Omega}_{- \text {regular operation. }}$ However, the roll of minimal $\gamma_{\hat{\Omega}}^{\star}$-open sets in ${ }^{\gamma_{\hat{\Omega}}^{\star}}{ }_{\text {-locally finite }}$ space has been discussed.

Keywords : ${ }^{\gamma_{\hat{\Omega}}^{\star}}{ }_{\text {-open set, }} \gamma_{\hat{\Omega}}^{\star}{ }_{\text {-Interior, }}{ }^{\gamma_{\hat{\Omega}}^{\star}}{ }_{\text {-Closure, minimal }}$ $\gamma_{\hat{\Omega} \text {-open set, }}^{\star} \gamma_{\hat{\Omega}}^{\star}$-locally finite space.

\section{INTRODUCTION}

I In a topological space, the notion of minimal open sets had been introduced by Nakaoka and Oda[4] in 2001. In 2012, Lellis Thivagar et al.[1] introduced the class of $\hat{\Omega}_{\text {-closed sets }}$ in a space which is independent of closed sets. Recently, [3] operation on the class of $\hat{\Omega}_{\text {-open sets have been introduced }}$ and studied. In this paper, we introduce the class of ${ }^{\gamma_{\hat{\Omega}}^{\star}}$-open sets and investigate their basic properties in terms of it's closure. However, Nakaoka's idea of minimal open sets has been extended to $\gamma_{\hat{\Omega}}^{\star}$-open sets and some of its elementary properties have been derived. Moreover, the behaviour of minimal $\gamma_{\hat{\Omega}}^{\star}$-open sets in a $\gamma_{\hat{\Omega}}^{\star}$-locally finite space has been investigated.

\section{PRELIMINARIES}

Some definitions and results that are used in this paper have been given in this section. Always $X$ or $(X, \tau)$ denotes a topological space on which no separation axioms assumed,

Revised Manuscript Received on December 16, 2019

* Correspondence Author

K.Poorani*, Research Scholar, Department of Mathematics, V.V.Vanniaperumal college for women,Virudhunagar. Email: kannapadaas@gmail.com

S.M.Meenarani, Associate Professor, Department of Mathematics, V.V.Vanniaperumal college for women,Virudhunagar. Email smmeenarani@gmail.com

M.Anbuchelvi, Associate Professor, Department of Mathematics, V.V.Vanniaperumal college for women,Virudhunagar. Email: rsanbuchelvi@gmail.com unless otherwise stated. For any subset $A$ of $X$, the closure (res.interior) of $A$ is denoted by $c l(A)(\operatorname{res} \operatorname{int}(A))$.

Definition 2.1 [2] A subset $A$ of a topological space $(X, \tau)$ is called a semi-open set if $A \subseteq \operatorname{cl}(\operatorname{int}(A))$. $S O(X)$ denotes the set of all semi-open sets in $(X, \tau)$.It's complement is known as semi-closed set on $X$.

Definition 2.2 ([1], Definition 3.1) Let $(X, \tau)$ be a topological space. $A$ is said to be $\hat{\mathbf{\Omega}}_{\text {-closed set if }} \delta c l(A) \subseteq U$ when $A \subseteq U$, where $U$ is a semi-open subset of $X$. The complement of $\hat{\Omega}_{\text {-closed set is an }} \hat{\Omega}_{\text {-open set. The family of }}$ all $\hat{\Omega}_{\text {-closed sets in a space }}(X, \tau)$ is denoted by ${ }^{\tau_{\hat{\Omega}^{*}}}$ Also $\hat{\Omega} O(X, \tau)$ or $\hat{\Omega} O(X)$ (resp. $\hat{\Omega} C(X, \tau)$ or $\hat{\Omega} C(X))$ denotes the

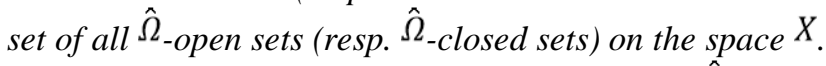

Definition 2.3 ([3], Definition 3.1) A function $\gamma: \hat{\Omega} O(X, \tau) \rightarrow$ $P(X)$ is called an operation on $\hat{\boldsymbol{\Omega}} \boldsymbol{O}(\boldsymbol{X}, \boldsymbol{\tau})$, if $U \subseteq \gamma(U)$ for every set $U \in \hat{\Omega} O(X, \tau)$. For any operation $\gamma, \gamma(X)=X$, and $\gamma(\varnothing)=\emptyset$.

Definition 2.4 ([3], Definition 3.3) A non-empty set $A$ of $X$ is called $\gamma_{\hat{\Omega}}$-open set if for each $x \in A$, there exists an $\hat{\Omega}$-open set $U$ such that $x \in U$ and $\gamma(U) \subseteq A$. The complement of ${ }^{\gamma_{\hat{\Omega}_{-}} \text {open set is a }}{ }^{\gamma_{\hat{\Omega}_{-}} \text {closed set. The set of all }}$ $\gamma_{\hat{\Omega}_{\text {-open }} \text { subsets of a topological space }}(X, \tau)$ is denoted by $\tau_{\gamma_{\hat{\Omega}} .}$

Definition 2.5 ([3], Definition 3.15) Let $(X, \tau)$ be a topological space. An operation $\gamma$ is said to be $\hat{\Omega}$ _regular if for every pair of sets $U, V \in \hat{\Omega} O(X, x)$, there exists an $\hat{\Omega}_{\text {-open set }} W_{\text {containing }} x_{\text {such that }} \gamma(W) \subseteq \gamma(U) \cap \gamma(V)$.

Remark 2.6 ([1], Remark 5.2) From the definition and Theorem 4.16, arbitrary intersection of an $\hat{\Omega}_{\text {-closed sets in a }}$ topological space $(X, \tau)$ is an $\hat{\Omega}_{\text {-closed set in }}(X, \tau), \hat{\Omega} c l(A)$

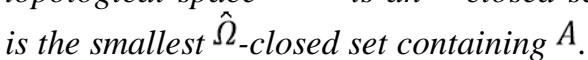

Theorem 2.7 ([3], Theorem 3.5) Arbitrary union of $\gamma_{\hat{\Omega}_{-o p e n}}$ sets is a $\gamma_{\hat{\Omega}_{-o p e n}}$ set in a topological space.

Proposition 2.8 ([3], Proposition 3.8) Every ${ }_{\hat{\Omega}_{-}}$open set is an $\hat{\Omega}_{\text {-open in a space }}$.

Proposition 2.9 ([3],

Proposition 3.17) Intersection 


\section{Remarks on $\gamma_{\Omega_{\text {-open sets and minimal }}^{\star}}^{\gamma_{\hat{\Omega}}^{\star}} \gamma_{\text {-open sets }}^{\star} \hat{\Omega}$}

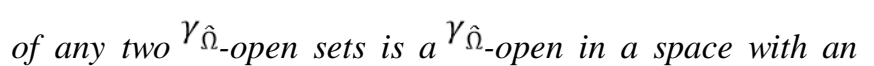
$\hat{\Omega}$-regular operation on $\hat{\Omega} O(X, \tau)$.

\section{III. $\gamma_{\hat{\Omega}-\text {-OPEN SETS }}^{\star}$}

Definition 3.1 A subset $A$ of a space $(X, \tau)$ is said to be a $\gamma_{\hat{\Omega} \_ \text {open }}^{\star}$ subset of $X$, if the following two axioms hold:

i) $A$ is a $\gamma_{\hat{\Omega}_{\text {-open }}}$ subset of $X$.

ii) For any $x \in A$, there exists an $\hat{\Omega}_{\text {-closed set }} F$ containing $x$ such that $F \subseteq A$.

The complement of a ${ }^{\gamma_{\hat{\Omega}}^{\star}}$-open set is a ${ }^{\gamma_{\hat{\Omega}}^{\star}}$-closed set in $X$. However, $\gamma_{\hat{\Omega}}^{\star} O(X)$ (resp. $\left.^{\gamma_{\hat{\Omega}}^{\star}} C(X)\right)$ denotes the set of all $\gamma_{\hat{\Omega}}^{\star}$-open sets (resp. $\gamma_{\hat{\Omega}}^{\star}$-closed sets) on $X$ and $\gamma_{\hat{\Omega}}^{\star} O(X, x)$ denotes the set of all $\gamma_{\hat{\Omega}}^{\star}$-open sets containing $x$.

Example3.2 Consider a space with $X=\{a, b, c, d\}$ and $\tau=\{\varnothing,\{a\},\{c\},\{a, b\},\{a, c\},\{a, b, c\},\{a, c, d\}, X\}$,

Define

$\gamma: \hat{\Omega} O(X, \tau) \rightarrow P(X)$

is defined

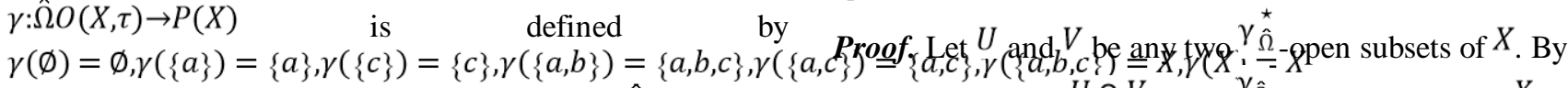

subset of $X$. Let $x \in U$ be arbitrary. Then $x \in U_{i}$ for some $i \in I$. Since $U_{i}$ is a $\gamma_{\hat{\Omega}}^{\star}$-open subset of $X$, there exist an $\hat{\Omega}$-closed set $F$ containing $x$ such that $F \subseteq U_{i} \subseteq U$. Therefore, $U$ is a $\gamma_{\hat{\Omega}}^{\star}$-open subset of $X$.

Remark 3.7. The intersection of any two ${ }^{\gamma_{\hat{\Omega}}^{\star}}$-open sets is not always a ${ }^{\gamma_{\hat{\Omega}}^{\star}}$-open set as seen from the following example.

Example 3.8. Let $X=\{a, b, c\}$ and $\tau=P(X)$. Define an operation $\gamma: \hat{\Omega} O(X) \rightarrow P(X)$ as follows:

$$
\gamma(W)= \begin{cases}W & \text { if } W=\{a, b\} \text { or }\{a, c\} \text { or } \emptyset \\ X & \text { Otherwise }\end{cases}
$$

$\gamma_{\hat{\Omega}}^{\star} O(X)=\{\varnothing,\{a, b\},\{a, c\}, X\}$. Here, the two sets $\{a, b\}$ and $\{a, c\}$ are ${ }^{\gamma_{\hat{\Omega}}^{\star}}$-open sets but $\{a\}$ is not a ${ }^{\gamma_{\hat{\Omega}}^{\star}}$-open in $X$.

Proposition 3.9. If $U$ and $V$ are ${ }^{\gamma_{\hat{\Omega}}^{\star}}$-open sets in a space $X$, then $U \cap V$ is a $\gamma_{\hat{\Omega}}^{\star}$-open set provided $\gamma$ is an $\hat{\Omega}_{\text {-regular }}$ operation.

.Here, $\gamma$ is an operation on $\hat{\Omega} O(X, \tau)$. Proposition 2.9, $U \cap V$ is a $\gamma_{\hat{\Omega}}$-open subset of $X$. Let $\tau_{\gamma_{\hat{\Omega}}}$

$x \in U \cap V$ be arbitrary. Since $U$ and $V$ are $\gamma_{\hat{\Omega}}^{\star}$-open subsets

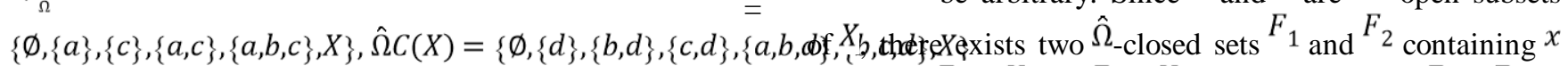
. Here, $\emptyset$ and $X$ are the only $\gamma_{\hat{\Omega}}^{\star}$-open subsets of $X$.

Proposition 3.3 In a space $(X, \tau)$, the following statements hold.
i) Every ${ }^{\gamma_{\hat{\Omega}}^{\star}}$-open subset is a ${ }^{\gamma_{\hat{\Omega}_{-}} \text {open set. }}$
ii) Every $\gamma_{\hat{\Omega}}^{\star}$-open set is an $\hat{\Omega}_{\text {-open set. }}$

Proof. ${ }^{i)}$ It follows straightly from the definition.

ii) It follows by combining $i$ ) and By Proposition 2.8

Remark 3.4 From the following example, it is seen that

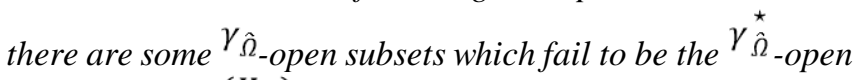
sets in a space $(X, \tau)$.

Example 3.5 Let $X=\{a, b, c\}, \tau=\{\emptyset,\{a\},\{a, b\}, X\}$; $\hat{\Omega} O(X)=\{\varnothing,\{a\}, X\} ; \hat{\Omega} C(X)=\{\varnothing,\{b, c\}, X\}$.

Define an operation $\gamma: \hat{\Omega} O(X) \rightarrow P(X)$ as follows:

$$
\gamma(W)= \begin{cases}W & \text { if } W=\{a\} \text { or } \varnothing \\ X & \text { Otherwise }\end{cases}
$$

Here, ${ }^{\gamma_{\hat{\Omega}}}=\{\varnothing,\{a\}, X\} ; \gamma_{\hat{\Omega}}^{\star} O(X)=\{\varnothing, X\}$.

$$
\text { Now }\{a\} \in \tau_{\gamma_{\hat{\Omega} b u t}}\{a\} \notin \gamma_{\hat{\Omega}}^{\star} O(X) \text {. }
$$

Theorem 3.6. Arbitrary union of ${ }^{\gamma_{\hat{\Omega}}^{\star}}$-open sets in a space $(X, \tau)$ is $a^{\gamma_{\hat{\Omega}}^{\star}}$-open set.

Proof. Let $\left\{U_{i / i} \in I\right\}$ be any collection of $\gamma_{\hat{\Omega}}^{\star}$-open sets in a space $X$ and let $U=\bigcup_{i \in I} U_{i}$. By Theorem 2.7, $U$ is a ${ }^{\gamma_{\Omega} \text {-open }}$ such that $F_{1} \subseteq U$ and $F_{2} \subseteq V$. By choosing $F$ as $F_{1} \cap F_{2}, F$ is an $\hat{\Omega}$-closed set containing $x$ such that $F \subseteq U \cap V$. Therefore, $U \cap V$ is a $\gamma_{\hat{\Omega}}^{\star}$-open subset of $X$.

$$
\gamma_{\hat{\Omega}}^{\star} \text {-Closure and } \gamma_{\hat{\Omega}}^{\star} \text {-Interior of a set have been }
$$
defined in the usual manner as follows:

Definition 3.10. $\gamma_{\hat{\Omega}_{\mathbf{\Omega}}}^{\star}$ closure of any subset ${ }^{A}$ of a space $(X, \tau)$ is denoted by ${ }^{\gamma_{\hat{\Omega}}^{\star}} \operatorname{cl}(A)$ and defined by $\gamma_{\hat{\Omega}}^{\star} \operatorname{cl}(A)=\bigcap\left\{F / F \in \gamma_{\hat{\Omega}}^{\star} C(X)_{\text {such that }} A \subseteq F\right\}$.

Always $\gamma_{\hat{\Omega}}^{\star} \operatorname{cl}(\varnothing)=\emptyset ; \gamma_{\hat{\Omega}}^{\star} \operatorname{cl}(X)=X ; \gamma_{\hat{\Omega}}^{\star} \operatorname{cl}(A)$ is a $\gamma_{\hat{\Omega}}^{\star}$-closed subset of $X$ and $\gamma_{\hat{\Omega}}^{\star}\left(\gamma_{\hat{\Omega}}^{\star} \operatorname{cl}(A)\right)=\gamma_{\hat{\Omega}}^{\star} \operatorname{cl}(A)$.

Definition 3.11. $\boldsymbol{\gamma}_{\hat{\Omega}_{-} \text {interior }}^{\star}$ of any subset $A_{\text {of a space }}$ $(X, \tau)$ is denoted by $\gamma_{\hat{\Omega}}^{\star} \operatorname{int}(A)$ and defined by $\gamma_{\hat{\Omega}}^{\star} \operatorname{int}(A)=\bigcup\left\{U / U \in \gamma_{\hat{\Omega}}^{\star} O(X)\right.$ such that $\left.U \subseteq A\right\}$. Always, $\gamma_{\hat{\Omega}}^{\star} \operatorname{int}(\varnothing)=\emptyset ; \gamma_{\hat{\Omega}}^{\star} \operatorname{int}(X)=X$ and $\gamma_{\hat{\Omega}}^{\star} \operatorname{int}(A)$ is a $\gamma_{\hat{\Omega}}^{\star}$-open subset of $X$.

Proposition 3.12. If $U$ and $V$ are any two subsets of a space $X$, then the following statements are true.

i) $U \subseteq V \Rightarrow \gamma_{\hat{\Omega}}^{\star} \operatorname{int}(U) \subseteq \gamma_{\hat{\Omega}}^{\star} \operatorname{int}(V)$.

ii) $U$ is $\gamma_{\hat{\Omega}}^{\star}$-open iff $U=\gamma_{\hat{\Omega}}^{\star} \operatorname{int}(U)$ 
iii) $U \subseteq V \Rightarrow \gamma_{\hat{\Omega}}^{\star} \operatorname{cl}(U) \subseteq \gamma_{\hat{\Omega}}^{\star} \operatorname{cl}(V)$.

iv) $U$ is $\gamma_{\hat{\Omega}}^{\star}$-closed iff $U=\gamma_{\hat{\Omega}}^{\star} \operatorname{cl}(U)$.

v) $X \backslash \gamma_{\hat{\Omega}}^{\star} \operatorname{int}(U)=\gamma_{\hat{\Omega}}^{\star} \operatorname{cl}(X \backslash U)$

vi) $X \backslash \gamma_{\hat{\Omega}}^{\star} c l(U)=\gamma_{\hat{\Omega}}^{\star} \operatorname{int}(X \backslash U)$.

Proof. It follows from the definition.

Proposition 3.13. For any point $x$ in a space $X$ and for any subset $A$ of $X,{ }^{x \in \gamma_{\Omega}^{\star}} \operatorname{cl}(A)$ iff $U \cap A \neq \emptyset$ for any $U \in \gamma_{\hat{\Omega}}^{\star} O(X, x)$

Proof. Assume that $x \in \gamma_{\hat{\Omega}}^{\star} c l(A)$ and there exists a $\gamma_{\hat{\Omega}}^{\star}$-open set $U$ containing ${ }^{x}$ such that $U \cap A=\varnothing$. Now, $X \backslash U$ is a $\gamma_{\hat{\Omega}}^{\star}$-closed set such that $A \subseteq X \backslash U$. That is, $X \backslash U \in\left\{F / F \in \gamma_{\hat{\Omega}}^{\star} C(X) ; A \subseteq F\right\} \quad$. In particular, $\gamma_{\hat{\Omega}}^{\star} \operatorname{cl}(A) \subseteq X \backslash U$, a contradiction to $x \in \gamma_{\hat{\Omega}}^{\star} \operatorname{cl}(A)$.

Conversely,on

contrary $x \notin \gamma_{\hat{\Omega}}^{\star} c l(A)=\bigcap\left\{F / F \in \gamma_{\hat{\Omega}}^{\star} C(X) ; A \subseteq F\right\}$. Then $x \notin F$ for some $\gamma_{\hat{\Omega}}^{\star}$-closed set $F$ such that $A \subseteq F$. If $U$ is chosen as $X \backslash F$, then $U$ is a $\gamma_{\hat{\Omega}}^{\star}$-open subset of $X$ such that $U \cap A=\varnothing$, a contradiction to hypothesis.

\section{MINIMAL ${ }^{\gamma_{\hat{\Omega}}}{ }_{\text {OPEN SETS AND }} \gamma_{\hat{\Omega}-\text { LOCALLY }}^{\star}$ FINITE SPACE}

Definition 4.1. $A^{\gamma_{\hat{\Omega}}^{\star}}$ - open subset $U$ of a space $X$ is said to be a minimal ${ }^{\star} \hat{\Omega}_{\text {-open set if }} \emptyset_{\text {and }} U$ are the only $\gamma_{\hat{\Omega}}^{\star}$-open subsets of $U$.

Example 4.2 Let $X=\{a, b, c\}$ and $\tau=P(X)=\hat{\Omega} O(X, \tau)$. Define an operation $\gamma: \hat{\Omega} O(X) \rightarrow P(X)$ as follows:

$$
\gamma(W)= \begin{cases}W & \text { if } W=\{a, b\} \text { or }\{a, c\} \text { or } \varnothing \\ X & \text { Otherwise }\end{cases}
$$

$\gamma_{\hat{\Omega}}^{\star} O(X)=\{\varnothing,\{a, b\},\{a, c\}, X\}=\gamma_{\hat{\Omega}} O(X)$. Here $\{a, b\}$ and $\{a, c\}$ are minimal ${ }^{\gamma_{\hat{\Omega}}^{\star}}$-open sets in $X$.

Proposition 4.3. Let $(X, \tau)$ be any topological space. If $U$ is a non-empty $\gamma_{\hat{\Omega}}^{\star}$-open subset of $X$ such that $\gamma_{\hat{\Omega}}^{\star} \operatorname{cl}(U)=\gamma_{\hat{\Omega}}^{\star} \operatorname{cl}(V)$ for any non-empty subset $V_{\text {of }} U$, then $U$ is a minimal $\gamma_{\hat{\Omega}}^{\star}$-open set.

Proof. On contrary, $U$ is assumed to be a non-minimal $\gamma_{\hat{\Omega}}^{\star}$-open subset of $X$ such that $\gamma_{\hat{\Omega}}^{\star} c l(U)=\gamma_{\hat{\Omega}}^{\star} c l(V)$ for any non-empty subset $V$ of $U$. By the definition of minimal open set, there exists a non-empty $\gamma_{\hat{\Omega}}^{\star}$-open set $W$ such that $W \varsubsetneqq U$. Choose $\quad x \in U \backslash W$. Now $W$ is a $\gamma_{\hat{\Omega}}^{\star}$-open set such that $x \notin W$.Therefore,
$W \cap \gamma_{\hat{\Omega}}^{\star} c l(\{x\})=\varnothing \quad$ or $\gamma_{\hat{\Omega}}^{\star} c l(\{x\}) \subseteq X \backslash \mathrm{W}$. Now $\{x\}$ is a non-empty subset of $U$. By hypothesis, $\gamma_{\hat{\Omega}}^{\star} c l(U)=\gamma_{\hat{\Omega}}^{\star} c l(\{x\}) \subseteq \mathrm{X} \backslash \mathrm{W}$

However,

$W \subsetneq \mathrm{U} \subseteq \gamma_{\hat{\Omega}}^{\star} c l(U)=\gamma_{\hat{\Omega}}^{\star} \operatorname{cl}(\{x\}) \subseteq \mathrm{X} \backslash \mathrm{W}$

$W \subseteq \mathrm{X} \backslash \mathrm{W}$, a contradiction.

Proposition 4.4. Let $\gamma$ be an $\hat{\Omega}_{\text {-regular operation in a space }}$ $X$.If $U$ is a minimal $\gamma_{\hat{\Omega}}^{\star}$-open set and $V$ is a $\gamma_{\hat{\Omega}}^{\star}$-open set then either $U \cap V=\emptyset_{\text {or }} U \subseteq V$.

Proof. Assume that $U \cap V=\emptyset$. By Proposition 3.9, $U \cap V$ is $a^{\gamma_{\hat{\Omega}}^{\star}}$-open set. By the definition of minimal $\gamma_{\hat{\Omega}}^{\star}$-open sets, $U \cap V=V$. Thus $U \subseteq V$.

Proposition 4.5. Let $\gamma$ be an $\hat{\Omega}_{\text {-regular operation in a space }}$ $X$. If $U$ and $V$ are any two minimal $\gamma_{\hat{\Omega}}^{\star}$-open sets, then either $U \cap V=\emptyset_{\text {or }} U=V$

Proof. Assume that $U \cap V=\varnothing$. Apply Proposition 4.4 for both minimal $\gamma_{\hat{\Omega}}^{\star}$-open sets $U$ and $V$. Then $U=V$.

Proposition 4.6. Let $\gamma$ be an $\hat{\Omega}_{\text {-regular operation in a space }}$ $X$ and $U$ be a minimal $\gamma_{\hat{\Omega}}^{\star}$-open set of $X$. Then for any $x \in U$, $U \subseteq V$ where $V$ is any $\gamma_{\hat{\Omega}}^{\star}$-open set containing $x$.

Proof. Assume the contrary that there exists $x \in U$ such that $U \nsubseteq V$ for any $\gamma_{\hat{\Omega}}^{\star}$-open subset $V$ containing $x$. By Proposition 3.9, $U \cap V$ is a $\gamma_{\hat{\Omega}}^{\star}$-open subset of $X$. As $x \in U \cap V, U \cap V \neq \varnothing$ such that $U \cap V \subseteq U$, a contradiction to $U$ is a minimal $\gamma_{\hat{\Omega}}^{\star}$-open subset.

Proposition 4.7. If $U$ is a minimal $\gamma_{\hat{\Omega}}^{\star}$-open subset of a space $X$, then $U=\bigcap_{x \in U}\left\{V / V \in \gamma_{\hat{\Omega}}^{\star} O(X, x)\right\}$.

Proof. By Proposition 4.6, for any $x \in U, U \subseteq V$ for any $\gamma_{\hat{\Omega}}^{\star} \quad$-open subset $\quad V \quad$ containing $\quad x \quad$. Then $U \subseteq \bigcap\left\{V / V \in \gamma_{\hat{\Omega}}^{\star} O(X, x)\right\} \quad$. In particular,

$\bigcap\left\{V / V \in \gamma_{\hat{\Omega}}^{\star} O(X, x)\right\} \subseteq U$

$U=\bigcap\left\{V / V \in \gamma_{\hat{\Omega}}^{\star} O(X, x)\right\}$

.Now

Proposition 4.8. Let $\gamma$ be an $\hat{\Omega}_{\text {-regular operation of a space }}$ $X$. If $U$ is a minimal ${ }_{\hat{\Omega}}^{\star}$-open subset of $X$, then for any $x \in X \backslash U$, and for any $\gamma_{\hat{\Omega}}^{\star}$-open set $V$ containing $x$, either $V \cap U=\emptyset_{\text {or }} U \subseteq V$.

Proof. It follows from Proposition 4.4.

Proposition 4.9. Let $\gamma$ be an $\hat{\Omega}_{\text {-regular operation in a space }}$ $X$. If $U$ is a non-empty minimal $\gamma_{\hat{\Omega}}^{\star}$-open subset of $X$, then $U \subseteq \gamma_{\hat{\Omega}}^{\star} c l(V)$ for any non-empty subset $V$ of $U$.

Proof. Let $x \in U$ be arbitrary and $W$ be any $\gamma_{\hat{\Omega}}^{\star}$-open set 


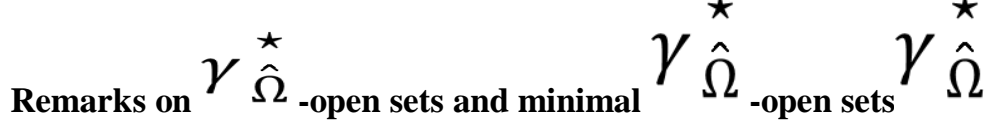

containing $x$. By Proposition 4.4, either $U \cap W=\emptyset$ or $U \subseteq W$. Since $x \in U \cap W$, the only possibility is $U \subseteq W$. Then, $V=U \cap V \subseteq W \cap V$. As $V \neq \emptyset, W \cap V \neq \emptyset$. Вy Proposition $3.13,{ }^{x \in \gamma_{\Omega}^{\star}} c l(V)$.

Characterization of minimal $\gamma_{\hat{\Omega}}^{\star}$-open set in a space is given as follows.

Theorem 4.10. Let $\gamma$ be an $\hat{\Omega}_{\text {-regular operation in a space }}$ $X$ and $U$ be any non-empty ${ }^{\gamma_{\hat{\Omega}}^{*}}{ }_{\text {-open }}$ subset of $X$. Then the following statements are equivalent:

i) $U$ is a minimal $\gamma_{\hat{\Omega}}^{\star}$-open set.

ii) $U \subseteq \gamma_{\hat{\Omega}}^{\star} c l(V)$ for any non-empty subset $V$ of $U$. iii) $\gamma_{\hat{\Omega}}^{\star} \operatorname{cl}(U)=\gamma_{\hat{\Omega}}^{\star} \operatorname{cl}(V)$ for any non-empty subset $V$ of $U$.

Proof. $i) \Rightarrow i i$ ) It follows from Proposition 4.9.

ii) $\Rightarrow$ iii) Let $V$ be any non-empty subset of $U$. By hypothesis $U \subseteq \gamma_{\hat{\Omega}}^{\star} \operatorname{cl}(V)$ and

hence

$\gamma_{\hat{\Omega}}^{\star} c l(U) \subseteq \gamma_{\hat{\Omega}}^{\star}\left(\gamma_{\hat{\Omega}}^{\star} c l(V)\right)=\gamma_{\hat{\Omega}}^{\star} \operatorname{cl}(V)$. On the other hand, $V \subseteq U \Rightarrow \gamma_{\hat{\Omega}}^{\star} c l(V) \subseteq \gamma_{\hat{\Omega}}^{\star} c l(U)$

iii) $\Rightarrow$ i) On contrary, $U$ is assumed to be a non-minimal $\gamma_{\hat{\Omega}}^{\star}$-open subset. Then, a non-empty ${ }^{\star} \hat{\Omega}_{\text {-open }}^{\star}{ }^{*} V$ can be chosen such that $V \varsubsetneqq U$. Having chosen $x \in U \backslash V$,

$V \cap \gamma_{\hat{\Omega}}^{\star} c l(\{x\})=\varnothing$ and hence $\gamma_{\hat{\Omega}}^{\star} c l(\{x\}) \subseteq X \backslash V$. But by hypothesis, $\gamma_{\hat{\Omega}}^{\star} \operatorname{cl}(\{x\})=\gamma_{\hat{\Omega}}^{\star} c l(U)$.It is concluded that $V \subseteq U \subseteq \gamma_{\hat{\Omega}}^{\star} c l(U)=\gamma_{\hat{\Omega}}^{\star} c l(\{x\}) \subseteq X \backslash V \quad$, a contradiction.

Therefore, $U$ is a minimal $\gamma_{\hat{\Omega}}^{\star}$-open set.

Definition 4.11. A space $X_{\text {is said to be }} \boldsymbol{\gamma}_{\hat{\Omega}_{-}}^{\star}$ locally finite space, if for every $x \in X$ there exists a finite $\gamma_{\hat{\Omega}}^{\star}$-open set $U$ in $X_{\text {such that }} x \in U$. Every finite topological space is always $\gamma_{\hat{\Omega}}^{\star}$-locally finite space.

Proposition 4.12. Every non-empty finite ${ }^{\gamma_{\hat{\Omega}}^{\star}}$-open set in a space $X_{\text {contains some non-empty finite minimal }} \gamma_{\hat{\Omega}}^{\star}$-open set.

Proof. Let $V$ be any non-empty finite ${ }^{\gamma_{\hat{\Omega}}^{\star}}$-open set in $X$. If $V$ is a minimal $\gamma_{\hat{\Omega}}^{\star}$-open set in $X$, then it meets our requirement. Suppose that $V$ is not a minimal $\gamma_{\hat{\Omega}}^{\star}$-open set in $X$. Then, there exists a non-empty finite ${ }^{\gamma_{\hat{\Omega}}^{\star}}$-open set $W_{1}$ such that $W_{1} \subsetneq V$. If $W_{1}$ is a minimal $\gamma_{\hat{\Omega}}^{\star}$-open set, then the condition is satisfied. If not, by proceeding like this we would arrive at a sequence of non-empty finite $\gamma_{\hat{\Omega}}^{\star}$-open sets $W_{1}, W_{2}, \ldots W_{n . .}$.such that $\ldots W_{n} \ldots \varsubsetneqq W_{2} \varsubsetneqq W_{1} \varsubsetneqq V$. Since $V$ is finite, there exists $m \in \mathbb{N}$ such that $W_{m}$ is a minimal $\gamma_{\hat{\Omega}}^{\star}$-open set such that $W_{m} \subseteq V$. Hence the result holds.
Proposition 4.13. Let $\gamma$ be an $\hat{\Omega}$-regular operation in a $\gamma_{\hat{\Omega}}^{\star}$-locally finite space $X$. Then every non-empty $\gamma_{\hat{\Omega}}^{\star}$-open set in a space $X$ contains some non-empty finite minimal $\gamma_{\hat{\Omega}}^{\star}$-open set.

Proof. Let $V$ be any non-empty $\gamma_{\hat{\Omega}}^{\star}$-open subset of $X$. Then choose $x \in V$. By the definition of $\gamma_{\hat{\Omega}}^{\star}$-locally finite space, there exists a finite ${ }^{\gamma_{\hat{\Omega}}^{\star}}$-open set $U$ such that $x \in U$. Since $U$ is finite, $U \cap V$ is also finite. By Proposition 3.9, $U \cap V$ is a finite $\gamma_{\hat{\Omega}}^{\star}$-open set containing $x$. By Proposition 4.12, there exists non-empty finite minimal $\gamma_{\hat{\Omega}}^{\star}$-open set $W$ such that $W \subseteq U \cap V \subseteq V$

Proposition 4.14. Let $X$ be a topological space and $\gamma$ be an

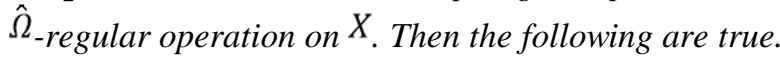

i) Let $V_{\alpha}, \alpha \in I$ be any $\gamma_{\hat{\Omega}}^{\star}$-open set and $U$ be a non-empty

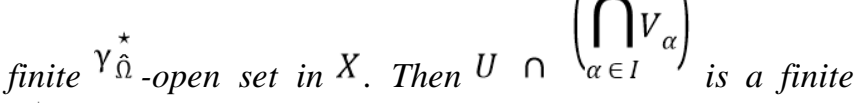
$\gamma_{\hat{\Omega}}^{\star}$-open set.

ii) Let $V_{\alpha}$ be $a^{\gamma_{\hat{\Omega}}^{\star}}$-open set for any $\alpha \in I$ and $V_{\beta}$ be a $\left(\bigcup_{V_{\beta}}\right)$ non-empty finite ${ }^{\gamma_{\hat{\Omega}}^{\star}}$-open set for any $\beta \in J$. Then $\beta \in J$<smiles>CC(C)O</smiles>
$\cap \underset{\alpha \in I}{n}$ is $a^{\gamma_{\hat{\Omega}}^{\star}}$-open set.

Proof. ${ }^{i)}$ Let $V_{\alpha}$ be a ${ }^{\star} \hat{\Omega}_{\text {-open subset for any }}^{\star} \alpha \in I$ and $U$ be a non-empty finite $\gamma_{\hat{\Omega}}^{\star}$-open set in $X$. Since $U$ is finite, $U \cap$ $\left(\bigcap_{\alpha \in I} V_{\alpha}\right)$

$\alpha \in I$ is a finite set. Then, there exists a positive integer $n$

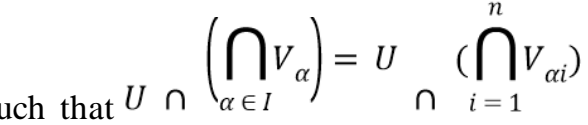
3.9, U $\cap\left(\bigcap_{\alpha \in I} V_{\alpha}\right)_{\text {is }} \gamma_{\hat{\Omega}}^{\star}$-open set.

ii) Let $U_{\alpha}$ be a $\gamma_{\hat{\Omega}}^{\star}$-open set for any $\alpha \in I$ and $V_{\beta}$ be a non-empty finite $\gamma_{\hat{\Omega}}^{\star}$-open set for any $\beta \in J$.By Theorem

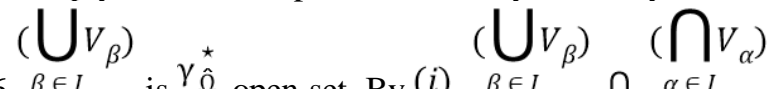

3.6, $\beta \in J$ is $\gamma_{\hat{\Omega}}^{\star}$-open set. By $(i), \beta \in J \cap{ }_{\alpha \in I}$

is $\gamma_{\hat{\Omega}}^{\star}$-open set in $X$.

\section{REFERENCES}

1. M.Lellis Thivagar, and M.Anbuchelvi, "Note on $\hat{\Omega}$-closed sets in topological spaces", Mathematical Theory and Modeling, 2012, pp.50-58.

2. N.Levine, "Semi-open sets and semi-continuity in topological spaces", Amer. Math. Monthly, 70,1, 1963, pp.36-41.

Published By:

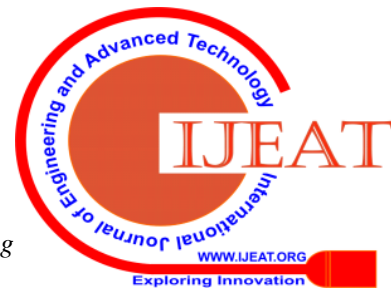


3. S.M.Meenarani, K.Poorani, and M.Anbuchelvi, "Operation on $\hat{\Omega}_{\text {-closed }}$ sets”, Malaya Journal of Matematik, Vol. S. No. 1, 2019, pp.7-11.

4. F.Nakaoka, and N.Oda, "Some applications of minimal open sets", Int. Math. Math. Sci. 27, 2001, No.8, pp.471-476.

5. S.F.Namiq, "New types of continuity and separation axioms based on operation in topological spaces", M.sc. Thesis, University of Sulaimani, 2011.

\section{AUTHORS PROFILE}

K. Poorani, Research Scholar,

Department of Mathematics, V. V. Vanniaperumal college for women, Virudhunagar. 